\title{
Treatment Group
}

National Cancer Institute

\section{Source}

National Cancer Institute. Treatment Group. NCI Thesaurus. Code C161322.

A study population that receives an intervention(s) within a trial. This could include the investigational product(s) or a comparator (e.g., placebo or an approved intervention). 